October 2, 2018

\title{
Spin-Orbit Force of Instanton-Induced Interaction in Strange and Charmed Systems
}

\author{
Sachiko Takeuchi \\ Department of Public Health and Environmental Science, \\ School of Medicine, Tokyo Medical and Dental University, \\ 1-5-45 Yushima, Bunkyo, Tokyo 113-8519, Japan
}

\begin{abstract}
Effects of the spin orbit-force on hadronic systems with strangeness or charm are investigated by a valence quark model with the instanton-induced interaction. By introducing this interaction, the spin-orbit splittings in the negative-parity hyperons becomes $0.14-$ 0.37 times smaller. The flavor-octet baryon mass spectrum and the splittings in the charmed baryons become consistent with the experiments. Though the splitting is also reduced in the flavor-singlet baryons, it still gives two third of the experimental value. The reduction comes from the cancellation between the one-gluon exchange and the instanton-induced interaction, which is channel-specific. In most of the two-baryon channels, the symmetric and antisymmetric spin-orbit force of the $\mathrm{YN}$ interaction remains strong after introducing this instanton effect. A few exceptional channels, however, are found where the cancellation affects strongly and the spin-orbit force becomes small.
\end{abstract}

PACS: 12.39.Jh, 13.75.Ev, 14.20.Jn, 14.20.Lq

Keywords: QCD instanton, spin-orbit force, hyperons, hyperon-nucleon interactions, quark model 


\section{Introduction}

Recent experiments on the systems with strangeness are making great progress. Especially the gamma spectroscopy has identified several gamma transitions, which give us valuable information on the spin part of the $\Lambda \mathrm{N}$ interaction [1]. From the observed levels of $\Lambda$-hypernuclei, it is believed that the spin-orbit force between $\Lambda$ and nucleon is very small comparing to that between two nucleons. It, however, is nontrivial to remove the nuclear effects to extract the interaction. Also, only the combined effect of the symmetric and antisymmetric spin-orbit force can be measured in the hypernuclei. Information on the noncentral parts of the YN interaction has not given directly from experiments yet. The theoretical investigation has been performed mainly by using the empirical YN interactions[2]. Here we employ a valence quark model to investigate the properties of the spin-orbit force in the strange systems. The quark model with the instanton-induced interaction, which is introduced in this work for the strange systems, is found to have an appropriate size and the channel dependence for the spin-orbit force and therefore will enable us to see its feature from a more fundamental viewpoint.

Valence quark models have been applied to low-energy light-quark systems and found to be successful in reproducing major properties of the hadrons and hadronic systems. These quark models usually contains three terms: the kinetic term, the confinement term, and the effective one-gluon exchange (OGE) term [3, 4, 5, 6, ,7,8]. It is considered that OGE stands for the perturbative gluon effects and that the confinement force represents the long-range nonperturbative gluon effects. We argue that a valence quark model should include the instanton-induced interaction (I I I) as a short-range nonperturbative gluon effect in addition to the other gluon effects[9, 10, 11, 12].

It is well known that the color magnetic interaction in OGE is responsible to produce many of the hadron properties. By adjusting the strength of OGE, the color magnetic interaction can reproduce the hyperfine splittings (e.g., ground state N- $\Delta$ mass difference) as well as the short-range repulsion of the two-nucleon systems in the relative $S$-wave 4 , [5, 6]. It, however, is also known that the strength of OGE, $\alpha_{s}$, determined in this empirical way is much larger than 1, which makes it hard to treat it as the perturbative effect.

The QCD instantons were originally introduced in relation to the $U_{A}(1)$ problem. It produces couplings of instantons to the surrounding light-quark zero modes[13, 14]. This leads a flavor-singlet interaction among quarks, which is believed to be an origin of the observed large mass difference of $\eta^{\prime}-\eta$ mesons. How these topological gluonic configurations 
behave in the actual vacuum has not been derived directly from QCD. A few empirical models with such interaction were proposed and found to reproduce the $\eta^{\prime}$ and $\eta$ meson masses and their properties 9, 15. Actually, several recent works indicate that the effects are also large in the baryon sector [9, 10, 11, 12, 16, 17, 18, 19. For example, the spin-spin part of I I I produces the nucleon- $\Delta$ mass difference [9,16] and the short-range repulsion between the two nucleons 99] as the color magnetic interaction. By introducing III we can reduces the empirical strength of OGE, $\alpha_{s}[9]$. Moreover, LS of I I I contributes the spin-orbit force in an interesting way[11, 12].

The valence quark model including only OGE as an origin of the spin-spin interaction has a spin-orbit problem. The spin-orbit part of OGE is strong; it is just strong enough to explain the observed large spin-orbit force between two nucleons [6]. On the other hand, the experimental mass spectrum of the excited baryons, $\mathrm{N}^{*}$ and $\Delta^{*}$ resonances, indicates that such a strong spin-orbit force should not exist between quarks 20]. A valence quark model in which the spin-orbit parts of the quark interaction are removed by hands can well simulate the observed mass spectrum [4,5, 21,22]. To explain both of the spin-orbit features at the same time is highly nontrivial.

In ref.[11], we demonstrated that introducing I I may solve the above difficulty in the nonstrange $P$-wave systems due to the channel-specific cancellation between OGE and III. Since here we consider the spin-orbit force on the $P$-wave systems, the quark pairs which are orbital-antisymmetric and spin-symmetric, i.e., only the pairs symmetric (or antisymmetric) simultaneously in the flavor and in the color spaces, are relevant. It is found that the contribution from the color- and flavor-symmetric quark pairs dominates in the two-nucleon force, while only color- and flavor-antisymmetric pairs exist in a baryon. Because OGE is vector-particle exchange, and III behaves like scalar-particle exchange, the sign of their spin-orbit parts is opposite to each other. Thus, where both of OGE and II contribute, namely for the color- and flavor-antisymmetric pairs, LS cancellation occurs. Therefore, it was expected that introducing II I would explain the strong LS in the two-nucleon systems and weak LS in the negative-parity baryons. In fact, we found such a channel-specific cancellation exists in the nonstrange $P$-wave systems. By introducing III strong enough to give the $\eta-\eta^{\prime}$ mass difference, LS in the single baryons almost vanishes. The above argument based on the symmetry holds even when the system is treated relativistic; we found the additional $\rho$-space does not change the conclusion qualitatively by employing a bag model.

We also investigated effects of the noncentral parts on the excited nonstrange baryons up to the principal number $=2$ and on the two-nucleon systems in a more quantitative way[12]. 
The discussion above is for the relative $P$-wave quark pairs; there is no such a cancellation between relative even pairs in the single baryons. One might wonder that the strong LS reappears in the excited positive baryons. It was found that that LS splittings becomes small even in the positive-parity baryons because major contribution comes from relative $P$-wave pairs even there. It was also found that LS between the two nucleons remains strong enough so that more realistic model, a quark cluster model, can actually fit the experimental data.

In this paper, we discuss effects of the spin-orbit part of III on the negative-parity hyperons and on the two-baryon systems with strangeness.

The negative-parity baryon mass spectrum by the present model shows that there is only weak spin-orbit force between the quarks due to the above cancellation. In the present choice of the parameters, the LS splittings becomes from 0.14 to 0.37 times smaller than that from the model only with OGE. The cancellation also occurs in the flavor-singlet baryons. There, however, seems to remain enough LS force that will give an appropriate splitting with the coupling to the $\mathrm{N} \bar{K}$ channel.

The symmetric and antisymmetric spin-orbit force between two baryons remains strong in most of the channels after introducing I I I as was found in the two-nucleon system, because the cancellation is weak. There, however, are a few exceptional channels where OGE-III LS cancellation also gives notable effects: the symmetric spin-orbit force of the $\mathrm{N} \Sigma(I=1 / 2)$ and of the $\mathrm{N} \Lambda-\mathrm{N} \Sigma$ channels almost vanishes after introducing III.

In section 2, we will explain the model we employed in this paper. The numerical results and discussions are in section 3. Summary are in section 4.

\section{Model}

We employed a valence quark model with a non-relativistic inter-quark potential. This model is chosen because the symmetry is clearly seen, and because $\mathrm{q}^{3}$ systems can be connected directly to $\mathrm{q}^{6}$ systems, both of which play essential roles in the discussion in this paper.

\subsection{Interaction}

We introduce II I as a short-range nonperturbative gluon effect in addition to OGE and the confinement potential. The model hamiltonian for quarks can be written as follows 9, 10, 11, 12, 23, :

$$
H_{\text {quark }}=K+\left(1-p_{\mathrm{III}}\right) V_{\mathrm{OGE}}+p_{\mathrm{III}} V_{\mathrm{III}}+V_{\text {conf }}
$$


where $V_{\mathrm{OGE}}$ and $V_{\mathrm{III}}$ are the Galilei invariant terms of the III and OGE potentials. The parameter $p_{\text {III }}$ represents the relative strength of the spin-spin part of I I I to OGE. It corresponds to the rate of the contribution from II I to the $S$-wave N- $\Delta$ mass difference, $\Delta M_{\mathrm{N} \Delta}$. When one introduces the interaction strong enough to give the observed $\eta-\eta^{\prime}$ mass difference, 408.7 MeV, $p_{\text {III }}$ becomes 0.3-0.4 [9, 10], which corresponds $90-120 \mathrm{MeV}$ of $\Delta M_{\mathrm{N} \Delta}$. As we will see later the spectra of the $P$-wave baryons including the flavor-singlet $\Lambda$ 's and those of $\Lambda_{c}$ 's also prefer this size of $p_{\mathrm{III}}$.

First, we derive the nonrelativistic potential for III. The effective interaction for III is 9, 10, 13, 16]:

$$
H_{\text {I I I }}=\sum_{i<j} V_{0}^{(2)}(i j) \bar{\psi}_{R}(i) \bar{\psi}_{R}(j)\left(1+\frac{3}{32} \lambda_{i} \cdot \lambda_{j}+\frac{9}{32} \lambda_{i} \cdot \lambda_{j} \sigma_{i} \cdot \sigma_{j}\right) \psi_{L}(j) \psi_{L}(i)+(\text { h.c. }),
$$

where $\psi_{R[L]}=\frac{1 \pm \gamma}{2} \psi, \lambda_{i}$ is the Gell-Mann matrix of the color $\mathrm{SU}(3)$ with $\lambda_{i} \cdot \lambda_{j}=\sum_{a=1}^{8} \lambda_{i}^{a} \lambda_{j}^{a}$, and $\sigma_{i}$ is the Pauli spin matrix for the $i$-th quark. $V_{0}^{(2)}(i j)$ is the strength of the two-body part of I I I, which survives only when quark $i$ and $j$ have different flavor from each other. The flavor dependence of $V_{0}^{(2)}(i j)$ can be found from its relation to the strength of the three-body part of III, $V_{0}$, for the set of quarks with the flavor $(i, j, k)=(\mathrm{u}, \mathrm{d}, \mathrm{s})$ :

$$
\begin{aligned}
V_{0}^{(2)}(i j) & =\frac{1}{2} V_{0}\left\langle\bar{\psi}_{k} \psi_{k}\right\rangle \\
& =\frac{1}{2} V_{0} \frac{m_{k}}{m_{u}}\langle\bar{u} u\rangle \\
& =\xi_{i} \xi_{j} \frac{1}{2} V_{0} \frac{m_{s}}{m_{u}}\langle\bar{u} u\rangle,
\end{aligned}
$$

where $m$ is the constituent quark mass, and $\xi_{j}=m_{u} / m_{j}$. Here, however, we take the twobody strength, $V_{0}^{(2)}(u d) \equiv V_{0}^{(2)}$ as a parameter determined later by eq. (13), and use the flavor dependence of the strength in the above relation.

At the flavor $\mathrm{SU}(3)$ limit, eq. (2) can be rewritten as below 9]. In this form it is more easily seen that this interaction operates only on flavor-antisymmetric quark pairs.

$$
\left.H_{\text {I I I }}=\sum_{i<j} V_{0}^{(2)}(i j) \bar{\psi}_{R}(i) \bar{\psi}_{R}(j) \frac{15}{8} \mathcal{A}_{i j}^{\text {flavor }}\left(1-\frac{1}{5} \boldsymbol{\sigma}_{i} \cdot \boldsymbol{\sigma}_{j}\right) \psi_{L}(j) \psi_{L}(i)+\text { (h.c. }\right),
$$

where $\mathcal{A}_{i j}^{\text {flavor }}=\left(1-P_{i j}^{\text {flavor }}\right) / 2$ is the antisymmetrizer in the flavor space.

We perform the nonrelativistic reduction to the lowest non-vanishing order in $(p / m)$ for each operator of different spin structure. The following potential between quarks of different masses is obtained:

$$
V_{\text {II }}=V_{0}(i j)\left[\left(1+\frac{3}{32} \lambda_{i} \cdot \lambda_{j}+\frac{9}{32} \lambda_{i} \cdot \lambda_{j} \sigma_{i} \cdot \sigma_{j}\right)\right.
$$




$$
\begin{aligned}
+ & \left(1+\frac{3}{32} \lambda_{i} \cdot \lambda_{j}\right)\left\{-\frac{1}{4 m_{i}^{2}} i\left(\sigma_{i} \cdot\left[q \times p_{i}\right]\right)+\frac{1}{4 m_{j}^{2}} i\left(\sigma_{j} \cdot\left[q \times p_{j}\right]\right)\right\}+\frac{9}{32} \lambda_{i} \cdot \lambda_{j}\{ \\
& \left.\frac{1}{4 m_{i}^{2}} i\left(\sigma_{j} \cdot\left[q \times p_{i}\right]\right)-\frac{1}{4 m_{j}^{2}} i\left(\sigma_{i} \cdot\left[q \times p_{j}\right]\right)+\frac{1}{2 m_{i} m_{j}}\left(i\left(\sigma_{i} \cdot\left[q \times p_{i}\right]\right)-i\left(\sigma_{j} \cdot\left[q \times p_{j}\right]\right)\right)\right\} \\
+ & \left(1+\frac{3}{32} \lambda_{i} \cdot \lambda_{j}\right)\left\{-\frac{1}{4 m_{i} m_{j}}\left(\sigma_{i} q\right)\left(\sigma_{j} q\right)\right\}+\frac{9}{32} \lambda_{i} \cdot \lambda_{j}\left\{\frac{1}{8}\left(\frac{1}{m_{i}^{2}}+\frac{1}{m_{j}^{2}}\right)\left(\sigma_{i} q\right)\left(\sigma_{j} q\right)\right. \\
& \left.\left.-\frac{1}{2 m_{i}^{2}}\left(\sigma_{i} p_{i}\right)\left(\sigma_{j} p_{i}\right)-\frac{1}{2 m_{j}^{2}}\left(\sigma_{i} p_{j}\right)\left(\sigma_{j} p_{j}\right)+\frac{1}{m_{i} m_{j}}\left(\sigma_{i} p_{j}\right)\left(\sigma_{j} p_{i}\right)\right\}\right] .
\end{aligned}
$$

Or in a form where the spin-orbit part and antisymmetric spin orbit terms are gathered separately,

$$
\begin{aligned}
V_{\text {I I I }} & =V_{0}^{(2)} \xi_{i} \xi_{j}\left[\left(1+\frac{3}{32} \lambda_{i} \cdot \lambda_{j}+\frac{9}{32} \lambda_{i} \cdot \lambda_{j} \sigma_{i} \cdot \sigma_{j}\right)\right. \\
& +\left\{\frac{9}{32} \lambda_{i} \cdot \lambda_{j}\left(\frac{1}{16}\left(\xi_{i}^{2}+\xi_{j}^{2}\right)+\frac{1}{4} \xi_{i} \xi_{j}\right)\right\} \frac{1}{m_{u}^{2}}\left(\sigma_{i}+\sigma_{j}\right) i\left[q \times \boldsymbol{p}_{i j}\right] \\
& +\left\{\left(1+\frac{3}{32} \lambda_{i} \cdot \lambda_{j}+\frac{9}{32} \lambda_{i} \cdot \lambda_{j}\right) \frac{1}{16}\left(\xi_{i}^{2}-\xi_{j}^{2}\right)\right\} \frac{1}{m_{u}^{2}}\left(\sigma_{i}-\sigma_{j}\right) i\left[q \times \boldsymbol{p}_{i j}\right] \\
& +\left\{\left(1+\frac{3}{32} \lambda_{i} \cdot \lambda_{j}\right)\left(-\frac{1}{4} \xi_{i} \xi_{j}\right)+\frac{9}{32} \lambda_{i} \cdot \lambda_{j} \frac{1}{8}\left(\xi_{i}^{2}+\xi_{j}^{2}\right)\right\} \frac{1}{m_{u}^{2}}\left\{\left(\sigma_{i} q\right)\left(\sigma_{j} q\right)-\frac{1}{3}\left(\sigma_{i} \cdot \sigma_{j}\right) q^{2}\right\} \\
& \left.+\frac{9}{32} \lambda_{i} \cdot \lambda_{j}\left\{-\frac{1}{8}\left(\xi_{i}^{2}+\xi_{j}^{2}\right)-\frac{1}{4} \xi_{i} \xi_{j}\right\} \frac{1}{m_{u}^{2}}\left\{\left(\sigma_{i} \boldsymbol{p}_{i j}\right)\left(\sigma_{j} \boldsymbol{p}_{i j}\right)-\frac{1}{3}\left(\sigma_{i} \cdot \sigma_{j}\right) \boldsymbol{p}_{i j}^{2}\right\}\right],
\end{aligned}
$$

where $\boldsymbol{q}$ is the three momentum transfer, $\boldsymbol{p}_{i j}=\left(m_{j} p_{i}-m_{i} p_{j}\right) /\left(m_{i}+m_{j}\right)$. The first line of eq. (8) is the central part, the second is the symmetric spin-orbit part, the third is the antisymmetric spin-orbit part, and the last two lines are the tensor part. The flavor symmetry is not obvious in eq. (8); when $m_{u}=m_{d}=m_{s}$, however, the interaction vanishes for flavorsymmetric pairs for each central, spin-orbit, and tensor part. The same procedure for the one-gluon exchange (OGE) interaction leads [7,8,8,24]

$$
\begin{aligned}
V_{\mathrm{OGE}} & =4 \pi \alpha_{s} \frac{\lambda_{i} \cdot \lambda_{j}}{4} \frac{1}{q^{2}}\left[1-\frac{1}{8}\left(\frac{1}{m_{i}^{2}}+\frac{1}{m_{j}^{2}}\right) q^{2}-\frac{1}{m_{i} m_{j}} p_{i} \cdot p_{j}-\frac{1}{4 m_{i} m_{j}}\left(\sigma_{i} \cdot \sigma_{j}\right) q^{2}\right. \\
& +\frac{1}{4 m_{i}^{2}} i\left(\sigma_{i} \cdot\left[q \times p_{i}\right]\right)-\frac{1}{4 m_{j}^{2}} i\left(\sigma_{j} \cdot\left[q \times p_{j}\right]\right)-\frac{1}{2 m_{i} m_{j}}\left(i\left(\sigma_{i} \cdot\left[q \times p_{j}\right]\right)-i\left(\sigma_{j} \cdot\left[q \times p_{i}\right]\right)\right) \\
& \left.+\frac{1}{4 m_{i} m_{j}}\left(\sigma_{i} q\right)\left(\sigma_{j} q\right)\right] .
\end{aligned}
$$

Or,

$$
\begin{aligned}
V_{\mathrm{OGE}} & =4 \pi \alpha_{s} \frac{\lambda_{i} \cdot \lambda_{j}}{4} \frac{1}{q^{2}}\left[1-\frac{1}{6 m_{u}^{2}} \xi_{i} \xi_{j}\left(\sigma_{i} \cdot \sigma_{j}\right) q^{2}\right. \\
& +\frac{1}{8 m_{u}^{2}}\left(\xi_{i}^{2}+\xi_{j}^{2}+4 \xi_{i} \xi_{j}\right)\left(\sigma_{i}+\sigma_{j}\right) \cdot i\left[q \times \boldsymbol{p}_{i j}\right]+\frac{1}{8 m_{u}^{2}}\left(\xi_{i}^{2}-\xi_{j}^{2}\right)\left(\sigma_{i}-\sigma_{j}\right) \cdot i\left[q \times \boldsymbol{p}_{i j}\right] \\
& \left.+\frac{1}{4 m_{u}^{2}} \xi_{i} \xi_{j}\left\{\left(\sigma_{i} q\right)\left(\sigma_{j} q\right)-\frac{1}{3}\left(\sigma_{i} \cdot \sigma_{j}\right) q^{2}\right\}\right]
\end{aligned}
$$


Note that, with $V_{0}^{(2)}$ negative, spin-spin terms of $V_{\text {OGE }}$ and $V_{\text {II I }}$ have the same sign while that spin-orbit parts have opposite sign.

The central spin-independent part of the interaction $H_{\text {quark }}$ in eq. (1) has the kinetic term, the confinement term, in addition to the central parts of $V_{\text {III }}$ and $V_{\text {OGE }}$. Once we restrict the model wave function to be $(0 s)^{n-1}(0 p)$ as we will do in this work, however, those central terms only give a constant mass shift for the all the states considered here. Also, we focus our attention to the spin-orbit force and neglect the tensor part of the interaction. We will omit these terms in the followings of this paper.

As we mentioned before, III also has a three-body part, which survives only when it operates on a flavor-singlet set of three quarks. Moreover, it vanishes when operates on a color-singlet set of three quarks; namely, there is no contribution for the single baryons considered here. The three-body term may play an important role in two-baryon systems. In fact, it is reported that contribution of its central part to the $\mathrm{H}$ dihyperon is about 50 $\mathrm{MeV}[10]$. Though its spin-orbit part may also contribute to two-baryon systems, we do not consider the term here for simplicity.

\subsection{Wave functions}

The model wave functions employed here are essentially the same as those in the appendix of ref. [12]. For the single baryons, the three valence quarks are taken in the color-singlet state, the orbital wave function is a harmonic oscillator $(0 \mathrm{~s})^{2}(0 \mathrm{p})$ with the size parameter $b$, the spin of three quarks is $1 / 2$ or $3 / 2$. The base wave function of the flavor part is $\mathrm{SU}(3)$ singlet, octet, and decuplet, which are mixed when the quark mass difference is introduced. The wave functions in the flavor-octet [21] symmetry in addition to the singlet $\left[1^{3}\right]$ symmetry are summarized in table 1 . We do not take into account the orbital wave function deformation caused by the quark mass difference nor the interaction.

As for two-baryon systems, we use

$$
\Psi=\mathcal{A}_{6 q}\left\{\phi_{1} \phi_{2} \psi_{0 p}(\boldsymbol{R})\right\}
$$

where $\phi_{i}$ is wave function of the $i$-th baryon with a gaussian orbital wave function with a size parameter $b, \psi_{0 p}$ is the $0 p$ harmonic oscillator wave function of a size parameter $\sqrt{2 / 3} b$, and $\boldsymbol{R}=\left(\left(\boldsymbol{r}_{1}+\boldsymbol{r}_{2}+\boldsymbol{r}_{3}\right)-\left(\boldsymbol{r}_{4}+\boldsymbol{r}_{5}+\boldsymbol{r}_{6}\right)\right) / 3$. Again we do not take into account the deformation of $\psi$. The matrix element

$$
U(R=0)=\langle\Psi|V| \Psi\rangle-\sum_{i}^{2}\left\langle\phi_{i}|V| \phi_{i}\right\rangle
$$


corresponds to an adiabatic potential for the two baryons at relative distance, $R=0$, and expresses the size of the short-range interaction between the baryons.

\subsection{Parameters}

We use an empirical quark model, which contains several parameters originally. Their values in the non-strange sector are the same as in refs. 6, 9, 10,11, 12]. The up and down quark mass is $1 / 3$ of the nucleon mass; the size parameter $b$ is taken to be a little smaller than the real nucleon size reflecting that the observed baryon size has contribution from the meson cloud; $\alpha_{s}$ and $V_{0}^{(2)}$ are determined to give the ground state $\mathrm{N}-\Delta$ mass difference $\Delta M_{\mathrm{N} \Delta}$ :

$$
\frac{4}{3 \sqrt{2 \pi}} \frac{\alpha_{s}}{m_{u}^{2} b^{3}}=-\frac{9}{4 \sqrt{2 \pi}^{3}} \frac{V_{0}^{(2)}}{b^{3}}=293 \mathrm{MeV} \equiv \Delta M_{\mathrm{N} \Delta} ;
$$

$p_{\text {III }}$ is taken to be 0.4 , which is consistent with the strength which gives the $\eta^{\prime}-\eta$ mass difference. The strange quark mass ratio, $\xi_{s}=m_{u} / m_{s}$, is taken to give a correct grand state $\Lambda-\Sigma$ mass difference. That for the charm quark, $\xi_{c}=m_{u} / m_{c}$, is also determined from the $\Lambda_{c}-\Sigma_{c}$ mass difference. We use the same $\alpha_{s}$ for the strange baryons and the charmed baryons; the energy dependence of the OGE strength is neglected. The results here, including $p_{\text {III }}$ dependence, only depend on above parameters through $\Delta M_{\mathrm{N} \Delta}, m_{u} b$, and the quark mass ratio, whose values are listed in table 2 . The dependence on the values of $m_{u} b$ and $m_{u} / m_{s}$ is not very large, so the results do not change much provided that the parameters are taken so that eq. (13) holds.

When we draw the figure to compare the obtained spectrum to the experiments, the kinetic and the central part of the quark interaction, which give an overall mass shift, are substituted by the observed baryon mass average for each strangeness $0,-1$ and -2 system. They are weighted by spin and charge degeneracy over the experimentally established ("four stars") octet baryons which corresponds to the $P$-wave baryons 20: i.e., N(1535), N(1520), $\mathrm{N}(1650)$ and $\mathrm{N}(1675)$ for the nonstrange baryons, $\Lambda(1670), \Lambda(1690), \Lambda(1830), \Sigma(1670)$ and

$\Sigma(1775)$ for the strangeness -1 system, and $\Xi(1820)$, three stars but most established one, for the strangeness -2 system.

\section{$3 \quad$ Results and discussions}

\subsection{Single baryons}

Our results of mass spectrum of $P$-wave baryons are summarized in figure 1 . The mass levels expressed by stars (with square blocks if the error ranges are given) are the experimental 
values 20]. The levels expressed by horizontal bars are results by the present model with $p_{\text {III }}=0.4$ : i.e., $40 \%$ of the $S$-wave N- $\Delta$ mass difference are originated by III. We consider that overall feature of the mass spectrum is reproduced quite well by the present model. The spin-orbit force becomes weak due to the same reason as the nonstrange negative-parity baryons: OGE-I I I LS cancellation in the relative $P$-wave quark pairs.

The most remarkable change in the negative-parity single baryon system as the flavor space is extended from $\mathrm{SU}(2)$ to $\mathrm{SU}(3)$ is that there appear two flavor-singlet states, $\Lambda(1405)$ and $\Lambda(1520)$, in addition to other new octet baryons, $\Lambda^{*}, \Sigma^{*}$ and $\Xi^{*}$.

It is a long standing problem whether $\Lambda(1405)$, which lays below the $\mathrm{N} \overline{\mathrm{K}}$ threshold, should be considered as a three-quark state or a bound state of the $\mathrm{N} \overline{\mathrm{K}}$ system[20,25]. Recently, Isgur pointed out that it should be regarded as a three-quark state, based on the similarity of its mass spectrum to the charmed baryons', $\Lambda_{c}[25]$. This problem should be investigated by using a model which handles the three-quark states and the $\mathrm{N} \overline{\mathrm{K}}$ system in a consistent way 22,26]. We, however, use a valence quark model here and examine what can be said from the valence quark picture.

The problem of the flavor-singlet $\Lambda$ masses in the valence quark model prediction can be summarized into two points: one is that whether one can produce appropriate LS splitting between the two singlet $\Lambda$ particles, the other is if there is enough splitting between the singlet and the octet $\Lambda$ particles. The former point is directly related to the theme of this paper. The latter point relates the size of spin-spin interaction, the origin of $\Delta M_{\mathrm{N} \Delta}$.

In our model the LS strength is governed by $p_{\text {IIII }}$. The $p_{\text {III }}$ dependence of $\Lambda^{*}$ mass spectrum is shown in figures $2 \mathrm{a}$ ) and $2 \mathrm{~b}$ ). The figure $2 \mathrm{a})$ is for the flavor $\mathrm{SU}(3)$ limit $\left(\xi_{s}=1\right)$, while the flavor $\mathrm{SU}(3)$ is broken by the quark mass difference $\left(\xi_{s}=0.6\right)$ in figure $\left.2 \mathrm{~b}\right)$. Note that the spin-orbit splittings can be much smaller after III LS is introduced. When OGE alone causes the observed $\Delta M_{\mathrm{N} \Delta}\left(p_{\mathrm{III}}=0\right)$, the calculated mass splittings between, for example, spin doublet of octet $\Lambda^{*}$, i.e., $\Lambda(1670)$ and $\Lambda(1690)$, is $224 \mathrm{MeV}$. This strong LS is canceled by II I LS by the same mechanism as the nonstrange baryons. As $p_{\text {III }}$ increases, the total LS becomes smaller. At $p_{\mathrm{III}}=0.5$, there is almost no spin-orbit force between quarks. The present model with $p_{\mathrm{III}}=0.4$ gives $31 \mathrm{MeV}$ for the above mass difference. The LS splittings in the other channels are also reduced to from 0.14 to 0.37 times smaller values. Though the LS-splittings seem to be still larger than the experimental ones, the discrepancy is no more serious, especially comparing to the large experimental error ranges. The dependence of the LS-splittings on $p_{\mathrm{III}}$ is very strong; when $p_{\mathrm{III}}=0.42$, the above splitting becomes $23 \mathrm{MeV}$.

The present model with $p_{\text {III }}=0.4$ gives flavor-singlet states with mass $1470 \mathrm{MeV}$ for 
$J=1 / 2$ and $1550 \mathrm{MeV}$ for $J=3 / 2$. By changing $p_{\text {III }}=0$ to 0.4 , the splitting has become from $333 \mathrm{MeV}$ to $80 \mathrm{MeV}$; while that of the experiment is $115 \mathrm{MeV}$. The upper level is $30 \mathrm{MeV}$ higher than the experiment. Suppose there are other attractive forces affecting specifically to the singlet so that they are $30 \mathrm{MeV}$ more bound, then the level corresponding to $\Lambda(1405)$ becomes $1440 \mathrm{MeV}$; which is higher than the $\mathrm{NK}$ threshold only by $8 \mathrm{MeV}$. We can conclude that this model with $p_{\text {III }}=0.4$ can give bulk amount of the LS-splitting between the flavor-singlet baryons and that it can be the pole required to explain the low mass of $\Lambda(1405)$ when the $\mathrm{N} \overline{\mathrm{K}}$ channel is introduced [20,22, 26].

When $m_{s}=m_{u}$, the LS-splitting between $\Lambda(1405)$ and $\Lambda(1520)$ is proportional to the LS-splitting in the $P$-wave octet baryons; the matrix element is twice larger than that of the octet baryons, which holds irrespective of introduction of I I I. That means, if one omits the spin-orbit force between quarks by hands as the usual way, there is no spin-orbit splittings between the singlet $\Lambda$ 's.

The quark mass dependence of OGE and I I is different from each other. The mass spectrum with $m_{u} \neq m_{s}$ is different when one reduces OGE LS by hands instead of introducing III. Suppose the OGE spin-orbit term is weakened to 0.18 times smaller without introducing I I I so that the above splitting between $\Lambda(1670)$ and $\Lambda(1690)$ becomes again $31 \mathrm{MeV}$, then the splitting of the singlet $\Lambda$ 's becomes $54 \mathrm{MeV}$. The result with OGE-I I I cancellation, 80 $\mathrm{MeV}$, is closer to the experiment, $115 \mathrm{MeV}$. Other difference between these two interaction, which persists at the flavor SU(3) limit, is in the relation between the sizes of the LS splitting of the baryon mass and of the LS force of the two baryon systems and will be discussed in the next subsection.

The mass difference between the nonstrange $S$-wave octet baryons and the decuplet baryons, i.e., $\Delta M_{\mathrm{N} \Delta}$, is a fitting parameter in our model, which determines the combined strength of OGE and III. The origin of this mass difference is the color magnetic interaction of OGE and the spin-spin interaction of III. These terms also produce the octet-decuplet mass difference in the $S$-wave baryons with strangeness and the octet-decuplet and the singlet-octet mass difference in the $P$-wave baryons.

The obtained value of the octet-decuplet mass difference of $S$-wave $\Sigma^{*}$ particles $(\Sigma(1385)$ and $\left.\Sigma^{*}\right)$ is $176 \mathrm{MeV}$ in our model while the experimental one is $192 \mathrm{MeV}$. One can see that the present model can give appropriate size and flavor dependence for this mass difference.

As for the $P$-wave baryons, the above terms divide the mass levels into three when $m_{s}=m_{u}$ (see Figure $2 \mathrm{a}$ around $p_{\mathrm{III}}=0.5$ ). The highest one consists of the decuplet baryons and the octet baryons with the quark spin of $3 / 2$. The middle level consists of 
the octet baryons with the quark spin of $1 / 2$. The lowest one is the singlet baryons. The splittings between the highest and the middle level is the same as that between the middle and the lowest level; they are half of $\Delta M_{\mathrm{N} \Delta}$ independent from the value of $p_{\mathrm{III}}$. Actually the singlet-octet separation here is much smaller than the observed one. We do not discuss here the possible origin of the required additional attraction for the singlet baryons. Let us mention, however, that the $\mathrm{N} \overline{\mathrm{K}}$ channel has the same flavor symmetry as the "pentaquark state" and therefore may have an attractive effect from the quark interaction 23 as well as the mesonic effects. The analysis with coupling to the $\mathrm{N} \overline{\mathrm{K}}$ channel should be required for further consideration. Also, the observation of the decuplet particles will help to see the situation.

Recent experiments indicate that there are two "flavor-singlet" charmed baryons. The lower one is $\Lambda_{c}(2594)$ with spin $=1 / 2$. The spin of the upper one, $\Lambda_{c}(2627)$, is not determined yet; the decay mode, however, suggests that this state has spin 3/2 20]. Their splitting is $32.7 \mathrm{MeV}$. It is interesting to see if the present model gives an appropriate prediction for these two $\Lambda_{c}$ 's.

The nonrelativity of the model is more appropriate for these states; the flavor $\mathrm{SU}(3)$, however, is not valid. The charmed baryons is considered as systems of two light quarks and one charm quark rather than the flavor octet or singlet states. The state is more close to the eigenstate of the hamiltonian if they are classified as

$$
\begin{aligned}
& |\alpha\rangle=\mid u d(\operatorname{spin}=0, \text { isospin }=0, \text { relative } S \text {-wave }), c\rangle \\
& |\beta\rangle=\mid u d(\operatorname{spin}=1, \text { isospin }=0, \text { relative } P \text {-wave }), c\rangle .
\end{aligned}
$$

$|\alpha\rangle$ corresponds to the total angular momentum, $J=(1 / 2),(3 / 2)$ states while $|\beta\rangle$ corresponds to $J=(1 / 2)^{2},(3 / 2)^{2},(5 / 2)$ states.

In figure 2c), the $p_{\text {III }}$ dependence of the mass spectrum with $m_{c} \rightarrow \infty$ is shown. The solid line corresponds to the $J=1 / 2$ state. The dashed lines correspond to the $J=1 / 2$ and $3 / 2$ states, which are degenerated. The dot-dashed line correspond to the $J=3 / 2$ and $5 / 2$ states, which are also degenerated. The mass of the lowest level at $p_{\mathrm{III}}=0.4$ is taken to be the observed lowest one, i.e., $\Lambda_{c}(2594)$. At $p_{\mathrm{III}}=0$, there is large spin-orbit force between quarks. The LS force is canceled when $p_{\mathrm{III}}$ increases, as the $\Lambda_{s}$ case. It vanishes around again $p_{\text {III }}=0.5$, where $|\alpha\rangle$ (lower level) and $|\beta\rangle$ (upper level) are separated only by the spin-spin interaction.

The instanton induced interaction comes from the instanton-light-quark coupling. Thus, we suppose there is no I I between the light quarks and the charm quark. By fitting the 
observed $S$-wave $\Lambda_{c^{-}} \Sigma_{c}$ difference, $168.5 \mathrm{MeV}$, at $p_{\mathrm{III}}=0.4$, we obtain $\xi_{c}=m_{u} / m_{c}=0.23$. It corresponds to $m_{c}=1364 \mathrm{MeV}$; the value is reasonable considering that the current mass of the charm quark is $1.0-1.6 \mathrm{GeV}$. The model gives $40 \mathrm{MeV}$ for the octet-decuplet mass difference of $S$-wave $\Sigma_{c}$, while the experimental value is $64.5 \mathrm{MeV} 20$.

In figure $2 \mathrm{~d}$ ), we show the $p_{\text {III }}$ dependence of $\Lambda_{c}$ mass levels at $\xi_{c}=0.23$. Again, the mass of the lowest level at $p_{\text {III }}=0.4$ is taken to be the observed lowest one. The overall feature is similar to the fig $2 \mathrm{c}$ ). The two lowest levels are spin $1 / 2$ and $3 / 2$ for $p_{\text {III }}>0.3$ while both of them are spin $1 / 2$ for $p_{\mathrm{III}}<0.3$. The present model at $p_{\mathrm{III}}=0.4$ gives $34 \mathrm{MeV}$ for the separation between the lowest spin $1 / 2$ and $3 / 2$ states. The experimental value is $32.7 \mathrm{MeV}$; the result is remarkably well. As seen from the figure, this mass difference is insensitive to $p_{\text {III }}$ provided that $p_{\text {III }}>0.3$, where the second lowest state has spin $3 / 2$.

The wave function of each level gives an information about whether the strange quark should be treated as a light quark or a heavy quark. Though it may be too simplified question, it helps to see rough properties of the systems. When $\xi_{s}=0.6$, the flavor-singlet components of the obtained levels corresponding to $\Lambda(1405)$ and $\Lambda(1520)$ are 0.98 and 0.84 , respectively, while the components of $|\alpha\rangle$ are 0.63 and 0.85 , respectively. In the lowest flavor singlet state, the strange quark acts a light quark. Its behavior in the higher level is just in-between of light and heavy. It seems that as far as the mass of the $\Lambda$ is concern, we can treat strange quark as a light quark rather than the heavy quark. The effects of the mass difference in the relative momentum, $\boldsymbol{p}_{i j}$, is included here but it is found to be small and almost indistinguishable in the figure 2b). Naturally, the charm quark acts more like a heavy quark; the flavor singlet components of $\Lambda_{c}$ are 0.79 and 0.42 while the components of $|\alpha\rangle$ are 0.89 and 0.94 , respectively.

The spin-orbit force, which is our main concern in this paper, is a relativistic effect. A relativistic model should be used for the check. As seen in ref.[11], the same conclusion is derived from the mass levels of negative-parity $\mathrm{N}^{*}$ calculated by the bag model as the present non-relativistic model. We expect that the situation is similar for the systems with strange quarks.

\subsection{Discussion by the symmetry}

One of the reasons why valence quark models can be so successful is that the model space has an appropriate symmetry. The mechanism of the LS cancellation, which affects largely in the excited baryons while only at a small extent in the two-nucleon systems, is also clearly seen from the discussion on the symmetry [1],12]. Such discussion can also be performed for 
systems with strangeness; which indicates that the situation in the single baryons and most of the strange two-baryon channels is similar to the nonstrange systems. However, there are a few exceptional YN channels, where III LS affects as strong as OGE LS.

Let us consider the spin-orbit part of the quark interaction which is color-diagonal and operates on relative $P$-wave quark pairs. We neglect here the quark mass difference in the relative momentum because it gives very small effects. The interaction can be decomposed as:

$$
\begin{aligned}
\mathcal{O}_{\mathrm{qSLS}} & =\mathcal{O}_{\mathrm{qSLS}} \overline{\mathcal{P}}^{A}+\mathcal{O}_{\mathrm{qSLS}} \mathcal{P}^{S} \\
\mathcal{O}_{\mathrm{qALS}} & =\mathcal{O}_{\mathrm{qALS}} \overline{\mathcal{P}}^{A}+\mathcal{O}_{\mathrm{qALS}} \overline{\mathcal{P}}^{S}+\mathcal{O}_{\mathrm{qALS}} \mathcal{P}^{S}+\mathcal{O}_{\mathrm{qALS}} \mathcal{P}^{A}
\end{aligned}
$$

where

$$
\begin{aligned}
\overline{\mathcal{P}}^{A} & \equiv \mathcal{A}^{\text {orb }} \mathcal{S}^{\text {spin }} \mathcal{A}^{\text {color }} \mathcal{A}^{\text {flavor }} \\
\mathcal{P}^{S} & \equiv \mathcal{A}^{\text {orb }} \mathcal{S}^{\text {spin }} \mathcal{S}^{\text {color }} \mathcal{S}^{\text {flavor }} \\
\overline{\mathcal{P}}^{S} & \equiv \mathcal{A}^{\text {orb }} \mathcal{A}^{\text {spin }} \mathcal{A}^{\text {color }} \mathcal{S}^{\text {flavor }} \\
\mathcal{P}^{A} & \equiv \mathcal{A}^{\text {orb }} \mathcal{A}^{\text {spin }} \mathcal{S}^{\text {color }} \mathcal{A}^{\text {flavor }}
\end{aligned}
$$

with antisymmetrizers $\mathcal{A}$ 's and symmetrizers $\mathcal{S}$ 's. The operator with bar is for color antisymmetric pairs, which is relevant to the single baryons. Those for the flavor-antisymmetric (-symmetric) quark pairs are marked by $A(S)$. The subscript qSLS [qALS] stands for the quark spin-orbit interaction which is proportional to the $(\sigma \pm \sigma)$ operator. $\mathcal{O}_{\mathrm{qSLS}}$ corresponds to the second term in the interaction, eq. (8) and the third term in eq. (10), while $\mathcal{O}_{\text {qSLS }}$ corresponds to the third term in eq. (8) and the forth term in eq. (10).

The matrix elements of OGE LS and III LS by a color-antisymmetric and a symmetric quark pair are listed in table 3 for each qSLS or qALS term. AS [S] stands for the contribution from a color-antisymmetric [symmetric] quark pair, which corresponds to the first [second] term in eq. (16) or to the first two [last two] terms in eq. (17). The matrix elements of qSLS listed in table 3 are for a ud quark pair; those for a us or a ds quark pair can be obtained by multiplying the entry by $\xi_{s}$ for OGE and $\xi_{s}^{2}$ for III. Those for qALS are for a us or a ds quark pair.

First we consider the system at $m_{u}=m_{s}$, where only $\mathcal{O}_{\mathrm{qSLS}}$, i.e., the $(\sigma+\sigma)$ terms, are relevant. The noncentral term of III contains only flavor-antisymmetric component, $\mathcal{O}_{\mathrm{qSLS}} \overline{\mathcal{P}}^{A}$, while OGE has both of the components in eq. (16). Since OGE is vector-particle exchange and III is alike to scalar-particle exchange, their spin-orbit term has an opposite sign where both of OGE and II I exist. Thus, there is a spin-orbit cancellation for color- and 
flavor-antisymmetric quark pairs. The LS force in a single baryon, which can be obtained by multiplying the entry of the antisymmetric pair in table 3 by the number of quark pairs, three, becomes weak.

We have a new type of spin-orbit force for the two-baryon systems besides the usual LS. Since isospin of the $s$-quark is zero, $\mathrm{NY}^{3} P_{1}$ and $\mathrm{NY}^{1} P_{1}$ may have the same isospin; namely, they are mixed by the strong interaction even at the flavor SU(3) limit 27]. The term which causes this mixing is called anti-symmetric spin-orbit force (ALS), and is written by the baryon coordinates as

$$
V_{A L S}=\sum_{i<j} g_{i j}\left(\sigma_{i}-\sigma_{j}\right) \cdot\left[\boldsymbol{q} \times \boldsymbol{P}_{i j}\right]
$$

with the coupling constant $g_{i j}=-g_{j i}$.

The symmetric spin-orbit force in the quark interaction, qSLS, induces this anti-symmetric spin-orbit force for the baryons, ALS, in addition to the ordinary symmetric spin-orbit force (SLS). ALS derived from the quark interaction does not vanish at the flavor SU(3) limit and can be comparably strong to SLS.

To obtain the spin-orbit force in the two baryon systems, one needs to know the factors in addition to the size of matrix elements for each kind of quark pairs. Coefficients which should be multiplied to the matrix elements in table 3 are listed in table 4 for various two-baryon systems. For example, OGE contribution from the flavor and color-antisymmetric quark pairs in $\mathrm{NN}^{3} P_{1}$ SLS is $(3 / 2)(3 / 75) \Delta M_{\mathrm{N} \Delta}=18[\mathrm{MeV}]$, while that from the color-symmetric quark pairs in $\mathrm{N} \Lambda{ }^{3} P_{1}$ SLS is $(-3 / 2)\left(6+10 \xi_{s}\right) / 36 \Delta M_{\mathrm{N} \Delta}=-147[\mathrm{MeV}]$.

The coefficients for various strange systems listed in table 4 indicates that contribution from color-symmetric quark pairs dominates in most of SLS and ALS in the strange twobaryons systems. In the two-nucleon system, for example, it can be seen from the fact that the coefficient of $\mathrm{S}, 38 / 75$, is much larger than the coefficient of $\mathrm{AS}, 3 / 75$. There, the size of SLS or ALS we obtain with non-zero $p_{\text {III }}$ is approximately equals to the one obtained by reducing the strength of OGE to $\left(1-p_{\text {III }}\right)$. Note that, suppose one reduces the LS splitting of the single baryons by weakening OGE LS by hands, the size of LS here can be about 20 $\%$ of the full OGE value, which is much smaller than the present result, about $60 \%$.

The exceptions are SLS of $\mathrm{N} \Sigma(I=1 / 2)$ and $\mathrm{N} \Lambda-\mathrm{N} \Sigma$ channels. The contribution from the color-antisymmetric quark pairs are as large as the symmetric one in these channels, which means that II I LS gives large effects. The OGE-I I I LS cancellation plays important role there, and the LS force becomes also small similarly to the LS splittings in the single baryons. 
The numerical calculation was performed also at $m_{u} \neq m_{s}$, where $\mathcal{O}_{\mathrm{qALS}}$ as well as the quark mass dependence of $\mathcal{O}_{\text {qSLS }}$ should be taken into account. Actually, the factor of qALS in table 4 is not very small comparing to qSLS. However, the size of the matrix element of one pair is about ten times smaller as seen in table 3. Thus we expect that the results will change only by order of $\left(1-\xi_{s}\right)$ when the quark mass difference is taken into account, which is confirmed by the numerical calculation.

\subsection{Two-baryon systems}

We investigate $U_{\mathrm{SLS}[\mathrm{ALS}]}(R=0)$ defined by eq. (12), the adiabatic potential between the two baryons at zero relative distance. This value is considered to express the strength of the short-range interaction between the two baryons.

The numerical results at $\xi_{s}=1$ and 0.6 with and without III are listed in table 4 . The size of ALS is comparable to SLS in general. The size of both kinds of the spin-orbit forces depends strongly on the channels. SLS of $\mathrm{N} \Lambda$ and $\mathrm{N} \Sigma(\mathrm{I}=3 / 2)$ diagonal, ALS of $\mathrm{N} \Sigma{ }^{3} P_{1^{-}}{ }^{1} P_{1}$, and ALS of $\mathrm{N} \Lambda{ }^{1} P_{1}-\mathrm{N} \Sigma{ }^{3} P_{1}$ are as strong as SLS of NN. ALS of $\mathrm{N} \Sigma(\mathrm{I}=3 / 2){ }^{3} P_{1^{-}}{ }^{1} P_{1}$ is very small. Size of both kinds of the spin-orbit forces in the other channels are from $20 \%$ to $40 \%$ of NN SLS.

When $\xi_{s}$ changes from 1 to 0.6 , N $\Lambda$ SLS is reduced by about $25 \%$ in both of the $p_{\text {III }}=0$ and $p_{\text {III }}=0.4$ cases. SLS of N $\Lambda$-N $\Sigma$, ALS of $N \Sigma{ }^{3} P_{1^{-}}{ }^{1} P_{1}$, and ALS of N $\Lambda{ }^{1} P_{1}-\mathrm{N} \Sigma{ }^{3} P_{1}$ are reduced by about $10 \%$ at $p_{\text {III }}=0$. ALS of $\mathrm{N} \Lambda{ }^{3} P_{1}-\mathrm{N} \Sigma{ }^{1} P_{1}$ at $p_{\text {III }}=0$ is reduced by about $20 \%$ while ALS of $N \Lambda{ }^{3} P_{1^{-}}{ }^{1} P_{1}$ at $p_{\text {III }}=0.4$ increases by about $20 \%$. The spin-orbit force in other channels, however, are almost the same by changing $\xi_{s}$.

Introducing I I I changes the channel dependence of the spin-orbit force. It was reported that if the interaction between baryons holds the flavor $\mathrm{SU}(3)$, which corresponds to the $p_{\mathrm{III}}=0$ and $\xi_{s}=1$ case here, the channel dependence is determined only by the SU(3) symmetry [27]. The $U_{\mathrm{SLS}[\mathrm{ALS}]}(R)$ from qSLS at $p_{\mathrm{III}}=0$ and $\xi_{s}=1$ or 0.6 , were calculated and found to hold the above relation between the channels except for the factor from the norm kernel[27]. Since III affects color- and flavor-antisymmetric quark pairs selectively, this relation deviates when III is switched on. As we discussed in the previous subsection, there are channels where the contribution from the color-antisymmetric pairs is comparably important to the symmetric ones. In the $\mathrm{N} \Sigma(I=1 / 2)$ and the $\mathrm{N} \Lambda-\mathrm{N} \Sigma$ channels, we found that introducing III changes actually the relative strength of the spin-orbit force between the baryons considerably.

The adiabatic potential at $R>0$ looks like a gaussian with the range of about $1 \mathrm{fm}[0,27]$. 
Since the potential we are considering here is SLS or ALS between relative $P$-wave, the potential at $R>0$ will be more important. Moreover, when we treat the quarks dynamically by, e.g., a quark cluster model, the potential we should consider between baryons is not the adiabatic one but the RGM potential, which is highly nonlocal[6, [7,8]. We argue, however, as far as a relative strength of SLS or ALS to the NN SLS is concerned, the conclusion here holds even when one performs more sophisticated calculations.

The ALS term is also in the meson-exchange interaction 27]; it was found that the tensor couplings of the vector-meson exchange can produce ALS at the flavor SU(3) limit. Its channel dependence is determined by $\mathrm{SU}(3)$. When the $\mathrm{SU}(3)$ is broken, various meson exchange produce the ALS term. The meson-induced ALS seems much smaller than that of quarks, though the size of the meson coupling is not well known.27.

Experimentally, information on the YN spin-orbit force has been given only through the

level splittings in $\Lambda$ hypernuclei. The ${ }_{\Lambda}^{9} \mathrm{Be}$ data suggest the $\mathrm{N} \Lambda$ spin-orbit interaction is very small[1]. The level splittings, however, gives only the combined strength of SLS and ALS; the strong SLS and ALS obtained from the quark model may cancels each other. It was reported that the other effect such as the YN tensor interaction may reduce the splitting[2]. More investigations both from the theoretical and experimental sides are necessary to understand the spin properties of the systems with strangeness.

\section{Summary}

We investigate the effects of the quark interaction induced from the instanton-light-quark coupling on the spin-orbit force in the negative-parity hyperons mass spectrum and in various relative $P$-wave two-baryon systems with strangeness. The spin-orbit force of this instantoninduced interaction (III) affects only the color-antisymmetric ones among the relative $P$ wave quark pairs. It cancels the spin-orbit force from the one-gluon exchange (OGE) for those pairs. Thus there is OGE-III LS cancellation where the color-antisymmetric pairs play an important role.

We employ a valence quark model where the strength of III is determined empirically: its spin-spin part gives $40 \%$ of the ground state nucleon- $\Delta$ mass difference. The value is consistent with the strength which can give the $\eta^{\prime}-\eta$ mass difference. The negative-parity baryon mass spectrum by the present model shows that there is only weak spin-orbit force between the quarks due to the above cancellation. In the present choice of the parameter set, the LS splittings are from 0.14 to 0.37 times smaller than that of the model only with 
OGE. The splittings in the flavor-octet baryons becomes consistent with the experiments. The splitting between the flavor-singlet $\Lambda$ 's becomes about two third of the experimental value. There seems to remain enough LS force that it will give an appropriate splitting with the coupling to the $\mathrm{N} \bar{K}$ channel.

Most of the symmetric and the antisymmetric spin-orbit force between two baryons remains strong after introducing III as was found in the two-nucleon system, because the color-symmetric quark pairs play an important role there. There, however, are a few exceptional channels where OGE-III LS cancellation are also large: as a result, the symmetric spin-orbit force of the $\mathrm{N} \Sigma(I=1 / 2)$ and of the $\mathrm{N} \Lambda-\mathrm{N} \Sigma$ channels become small.

The conclusion will be more quantitative if one extends the model, e.g., to take into account the meson effects or the deformation of the relative motion wave functions between the baryons. The properties of the excited baryons and YN interaction should be investigated both in the theoretical and in the experimental way.

The author would like to thank K. Yazaki and M. Oka for valuable discussions. This work was supported in part by the Grant-in-Aid for scientific research Priority Areas (Strangeness Nuclear Physics) of the Ministry of Education, Science, Sports and Culture of Japan. 


\section{References}

[†] Electronic mail: sachiko@thaxp1.tanashi.kek.jp.

[1] W. Bruckner, et al., Phys. Lett. 79B(1978)157; H. Tamura, et al., Nucl. Phys. A479(1988)161c; M. Bedjidian, et al., Phys. Lett. 83B(1979)252; M. May, et al., Phys. Rev. Lett. 47(1981)1106; ibid. 51(1983)2085; ibid. 78(1997)4343;

[2] C. B. Dover and H. Feshbach, Ann. Phys. (N.Y.) 198(1990)321; ibid. 217(1992)51; E. Hiyama, M. Kamimura, T. Motoba, T. Yamada, and Y. Yamamoto, Phys. Rev. C53(1996)2075; H. Hiyama, et al., Proceedings of 1st SUT-KEK seminar (Apr, 1998).

[3] T. A. DeGrand and R. L. Jaffe, Ann. of Phys. 100(1976)425; T. A. DeGrand, Ann. of Phys. 101(1976)496.

[4] N. Isgur and G. Karl, Phys. Rev. D18(1978)4187.

[5] N. Isgur, In. J. of Mod. Phys., 1(1992)465 and references therein; G. Karl, In. J. of Mod. Phys., 1(1992)491.

[6] O. Morimatsu, K. Yazaki, and M. Oka, Nucl. Phys. A424(1984)412; S. Takeuchi, K. Shimizu, and K. Yazaki, Nucl. Phys. A504(1989)777; K. Shimizu, Rep. Prog. Phys. $\mathbf{5 2 ( 1 9 8 9 ) 1 ~ a n d ~ r e f e r e n c e s ~ t h e r e i n . ~}$

[7] O. Morimatsu, S. Ohta, K. Shimizu, and K. Yazaki, Nucl. Phys. A420(1984)573; Y. Koike, Nucl. Phys. A454(1986)509; M. Oka, Prog. Theor. Phys. Suppl. 120(1995)95.

[8] Y. Suzuki and K. T. Hecht, Nucl. Phys. A420(1984)525; Y. Fujiwara, Prog. Theor. Phys. 88(1992)933;Prog. Theor. Phys. 89(1993)455;Prog. Theor. Phys. 90(1993)105. Y. Fujiwara, C. Nakamoto, and Y. Suzuki Prog. Theor. Phys. 94(1995)215; ibid. 94(1995)353; Phys. Rev. C54(1996)2180; Phys. Rev. Lett. 76(1996)2242.

[9] M. Oka and S. Takeuchi, Nucl. Phys. A524(1991)649; Phys. Rev. Lett. 63(1989)1780.

[10] S. Takeuchi and M. Oka, Phys. Rev. Lett. 66(1991)1271.

[11] S. Takeuchi, Phys. Rev. Lett. 73(1994)2173.

[12] S. Takeuchi, Phys. Rev. D53(1996)6619.

[13] G. 't Hooft, Phys. Rev. D14(1976)3432; M.A. Shifman, A.I. Vainshtein and V.I. Zakharov, Nucl. Phys. B163(1980)46; E.V. Shuryak, Phys. Rep. C115(1984)151. 
[14] C. G. Callan, Jr, R. Dashen, and D. J. Gross, Phys. Rev. D17(1978)2717; C. G. Callan, Jr, R. Dashen, D. J. Gross, F. Wilczek, and A. Zee, Phys. Rev. D18(1978)4684.

[15] M. Takizawa and M. Oka, Phys. Lett. B359(1995)210; Phys. Lett. B(1996)in press(E).

[16] N.I. Kochelev, Sov. J. Nucl. Phys. 41(1985)291; E.V. Shuryak and J.L. Rosner, Phys. Lett. B218(1989)72.

[17] O. Morimatsu and M. Takizawa, Nucl. Phys. A554(1993)635.

[18] M. C. Chu, et al., Phys. Rev. D49(1994)6039;

[19] S. Chernyshev, M. A. Nowak, and I. Zahed, Phys. Rev. D53(1996)5176.

[20] C. Caso, et al., The European Physical Journal C3(1998)1

[21] J. Wroldson and F. Myhrer, Z. Phys. C25(1984)59; F. Myhrer and J. Wroldson, Z. Phys. C25(1984)281; Y. Umino and F. Myhrer, Phys. Rev. D39(1989)3391.

[22] M. Arima and K. Yazaki, Nucl. Phys. A506(1990)553; M. Arima, S. Matsui and K. Simizu, Phys. Rev. C49(1994)2831; T. Hamaie, M. Arima, and K. Masutani, Nucl. Phys. A591(1995)675.

[23] H. Hogaasen and P. Sorba, Nucl. Phys. B145(1978)119; H. J. Lipkin, Phys. Lett. 195B(1987)484; S. Takeuchi, S. Nussinov, and K. Kubodera, Phys. Lett. B318(1993)1.

[24] A. De Rujula, H. Georgi and S.L. Glashow, Phys. Rev. D12(1975)147.

[25] N. Isgur, Nucl. Phys. A623(1997)37c

[26] D. Kiang et al., Phys. Rev. C30(1984)1638.

[27] M. Oka, Nucl. Phys. A629(1998)379c; M. Oka and Y. Tani, in preparation. 
Figure 1: Negative-parity baryon mass spectrum.

Figure 2: The $p_{\text {III }}$ dependence of the $P$-wave $\Lambda^{*}$ mass.

Each graph corresponds to the case where a) quark mass ratio, $\xi_{s}=m_{u} / m_{s}=1$, b) $\left.\xi_{s}=0.6, \mathrm{c}\right)$ no interaction between the charm and other quarks, and d) $\xi_{c}=m_{u} / m_{c}=0.23$. In figures a), b) and d), solid lines stand for the spin 1/2 states, dotted lines for the spin $3 / 2$ states, and dot-dashed lines for the spin 5/2 states. Spin quantum numbers of the degenerated states are written in figure c). 
Table 1: Single baryon wave functions in the flavor space.

The SU(3) wave function in the mixed [anti]symmetric (MS[MA]) of the [21] symmetry and in the $\left[1^{3}\right]$ symmetry are given. For the [21] states with non-zero $z$-component of the isospin, the flavor of $q_{1}$ and $q_{2}$ and overall phase $(P)$ of the wave function $|\mathrm{MA}\rangle=$ $P \frac{1}{\sqrt{2}}\left(q_{1} q_{2}-q_{2} q_{1}\right) q_{1}$, and $|\mathrm{MS}\rangle=P \frac{1}{\sqrt{6}}\left(2 q_{1} q_{1} q_{2}-q_{1} q_{2} q_{1}-q_{2} q_{1} q_{1}\right)$ are listed. For the other states, coefficients of the $q_{1} q_{2} q_{3}$ components together with the normalization $n$ are listed.

\begin{tabular}{|c|c|c|c|c|c|c|c|c|}
\hline $\mathrm{B}$ & $q_{1}$ & $q_{2}$ & $P$ & & & & & \\
\hline $\mathrm{p}$ & $\mathrm{u}$ & $\overline{\mathrm{d}}$ & + & & & & & \\
\hline $\mathrm{n}$ & $\mathrm{d}$ & $\mathrm{u}$ & - & & & & & \\
\hline$\Sigma^{+}$ & $\mathrm{u}$ & $\mathrm{s}$ & - & & & & & \\
\hline$\Sigma^{-}$ & $\mathrm{d}$ & $\mathrm{S}$ & - & & & & & \\
\hline$\Xi^{0}$ & $\mathrm{~s}$ & $\mathrm{u}$ & + & & & & & \\
\hline$\Xi^{-}$ & $\mathrm{s}$ & d & + & & & & & \\
\hline \multicolumn{2}{|l|}{$\overline{\mathrm{B}}$} & $\frac{1}{2 \mathrm{uds}}$ & dus & $\mathrm{dsu}$ & usd & sud & $\mathrm{sdu}$ & $n^{-2}$ \\
\hline \multirow[t]{2}{*}{ 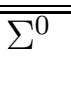 } & $\overline{\mathrm{MA}}$ & $\overline{0}$ & 0 & -1 & -1 & 1 & 1 & $\overline{4}$ \\
\hline & MS & -2 & -2 & 1 & 1 & 1 & 1 & 12 \\
\hline \multirow[t]{2}{*}{$\Lambda^{8}$} & MA & 2 & -2 & -1 & 1 & -1 & 1 & 12 \\
\hline & MS & 0 & 0 & -1 & 1 & 1 & -1 & 4 \\
\hline$\Lambda^{1}$ & A & 1 & -1 & 1 & -1 & 1 & -1 & 6 \\
\hline
\end{tabular}

Table 2: Model parameters.

\begin{tabular}{cccc}
\hline \hline$\Delta M_{\mathrm{N} \Delta}[\mathrm{MeV}]$ & $m_{u} b$ & $m_{u} / m_{s}$ & $m_{u} / m_{c}$ \\
\hline 293 & 0.79 & 0.6 & 0.23 \\
\hline \hline
\end{tabular}


Table 3: The spin-orbit matrix elements of two-baryon systems.

Matrix elements of OGE and II I by each color-antisymmetric (AS) and symmetric (S) quark pairs are listed in unit of the grand state N- $\Delta$ mass difference, $\Delta M_{\mathrm{N} \Delta}$. Those from the $(\sigma+\sigma)$ term in the quark potential, qSLS, are for ud quark pairs; for us or ds pairs, $\xi_{s}$ for OGE, $\xi_{s}^{2}$ for III should be multiplied. Those from the $(\sigma-\sigma)$ term, qALS, are for us or ds pairs. These matrix elements are to be multiplied by the factors listed in table 4 .

\begin{tabular}{ll|cc}
\hline \hline & & AS & S \\
\hline \multirow{2}{*}{ qSLS } & OGE & $3 / 2$ & $-3 / 4$ \\
& I I I & $-1 / m^{2} b^{2}$ & 0 \\
\hline \multirow{2}{*}{ qALS } & OGE & $\left(1-\xi_{s}^{2}\right) / 4$ & $-\left(1-\xi_{s}^{2}\right) / 8$ \\
& I I I & 0 & 0 \\
\hline \hline
\end{tabular}

Table 4: Symmetric and antisymmetric spin-orbit forces in the two-baryon systems.

Size of the SLS and ALS forces of various two-baryon systems are shown. Coefficients which should be multiplied to the entries in Table 3 are listed for each of the colorantisymmetric quark pair (AS) and the symmetric pair (S) and for each of ud and us quark pair, which is to be divided by the entry under $n^{-1}$. The obtained $U(R=0)$ in eq. (12) for each $m_{u} / m_{s}=1$ and 0.6, and the contribution of I I I, $p_{\text {III }}$, is 0 (OGE only) and 0.4 are listed separately in $\mathrm{MeV}$ together with the relative strength to NN SLS.

\begin{tabular}{|c|c|c|c|c|c|c|c|c|c|c|c|c|}
\hline & \multicolumn{4}{|c|}{ qSLS } & \multirow{3}{*}{\begin{tabular}{cc}
\multicolumn{2}{c|}{ qALS } \\
AS & S \\
us & us
\end{tabular}} & \multirow[b]{3}{*}{$n^{-1}$} & \multicolumn{5}{|c|}{$\overline{\overline{U(R=0)}}$} \\
\hline & & \multicolumn{2}{|l|}{$\mathrm{AS}$} & \multicolumn{2}{|l|}{$\mathrm{S}$} & & & \multicolumn{3}{|c|}{$m_{u} / m_{s}=1$} & \multicolumn{2}{|c|}{$m_{u} / m_{s}=0.6$} \\
\hline & & & & $\mathrm{ud}$ & us & & & $p_{\text {III }}=0$ & $p_{\text {III }}=$ & $=0.4$ & $p_{\text {III }}=0$ & $p_{\text {III }}=0.4$ \\
\hline \multicolumn{13}{|l|}{$\overline{\text { SLS }}$} \\
\hline$\overline{\mathrm{NN}-\mathrm{NN}}$ & \begin{tabular}{|lll}
2 & 1 & 1
\end{tabular} & 3 & & 38 & 0 & 0 & 75 & -94 & -61 & 1.00 & -94 & -61 \\
\hline $\mathrm{N} \Lambda$ & $\begin{array}{lll}1 & 1 & 1\end{array}$ & 1 & 1 & 6 & 10 & 2 & 36 & -73 & -51 & 0.83 & -54 & -37 \\
\hline $\mathrm{N} \Sigma-\mathrm{N} \Sigma$ & $\begin{array}{lll}1 & 1 & 1\end{array}$ & $69-$ & & 90 & -10 & $-18-42$ & 516 & $22-0.24$ & -3 & 0.05 & $22-0.23$ & 0.06 \\
\hline $\mathrm{N} \Lambda-\mathrm{N} \Sigma$ & $\begin{array}{lll}1 & 1 & 1\end{array}$ & $-3-$ & & 8 & 0 & $\begin{array}{ll}0 & -4\end{array}$ & $12 \sqrt{129}$ & -32 & -14 & 0.22 & 0.30 & -13 \\
\hline $\mathrm{N} \Sigma-\mathrm{N} \Sigma$ & $\begin{array}{lll}3 & 1 & 1 \\
\end{array}$ & 3 & 3 & 69 & 7 & $\begin{array}{ll}-9 & -3\end{array}$ & 150 & -94 & -61 & 1.00 & 1.02 & -61 \\
\hline \multicolumn{13}{|l|}{$\overline{\mathrm{ALS}}$} \\
\hline$\overline{\mathrm{N} \Lambda-\mathrm{N} \Lambda}$ & $\begin{array}{lll}1 & 1 & 0\end{array}$ & $1-$ & & 6 & -2 & 10 & 36 & -37 & -18 & 0.30 & -37 & $\begin{array}{ll}-23 & 0.38\end{array}$ \\
\hline $\mathrm{N} \Sigma-\mathrm{N} \Sigma$ & $\begin{array}{lll}1 & 1 & 0\end{array}$ & 9 & $18-$ & -102 & -6 & $1-10$ & $36 \sqrt{129}$ & $87-0.93$ & & -0.71 & $79-0.85$ & $43-0.69$ \\
\hline $\mathrm{N} \Lambda-\mathrm{N} \Sigma$ & $\begin{array}{lll}1 & 1 & 0\end{array}$ & $3-$ & & 14 & -2 & $\begin{array}{ll}3 & 10\end{array}$ & 108 & $\begin{array}{ll}-37 & 0.39\end{array}$ & -18 & 0.30 & $-30 \quad 0.32$ & -19 \\
\hline $\mathrm{N} \Lambda-\mathrm{N} \Sigma$ & $\begin{array}{lll}1 & 0 & 1\end{array}$ & 3 & 6 & -30 & -6 & $3-10$ & $12 \sqrt{129}$ & $87-0.93$ & 44 & -0.71 & $79-0.84$ & $42-0.69$ \\
\hline $\mathrm{N} \Sigma-\mathrm{N} \Sigma$ & $\begin{array}{lll}3 & 1 & 0\end{array}$ & -9 & 9 & -15 & 15 & $\begin{array}{ll}-1 & -5\end{array}$ & $90 \sqrt{5}$ & 0.00 & 0 & 0.00 & 0.01 & $3-0.05$ \\
\hline
\end{tabular}




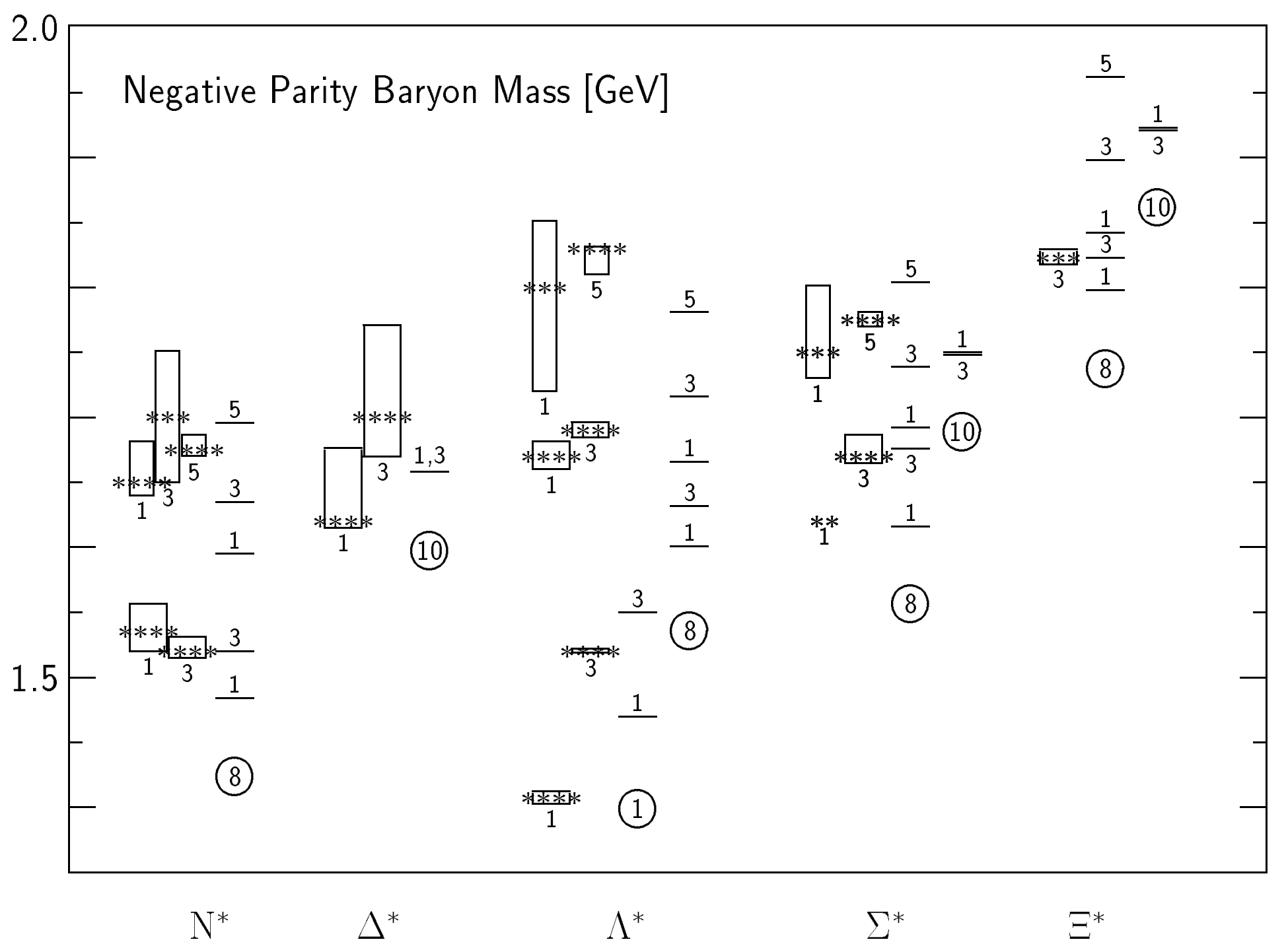

Fig 1 Negative-parity baryon mass spectrum 


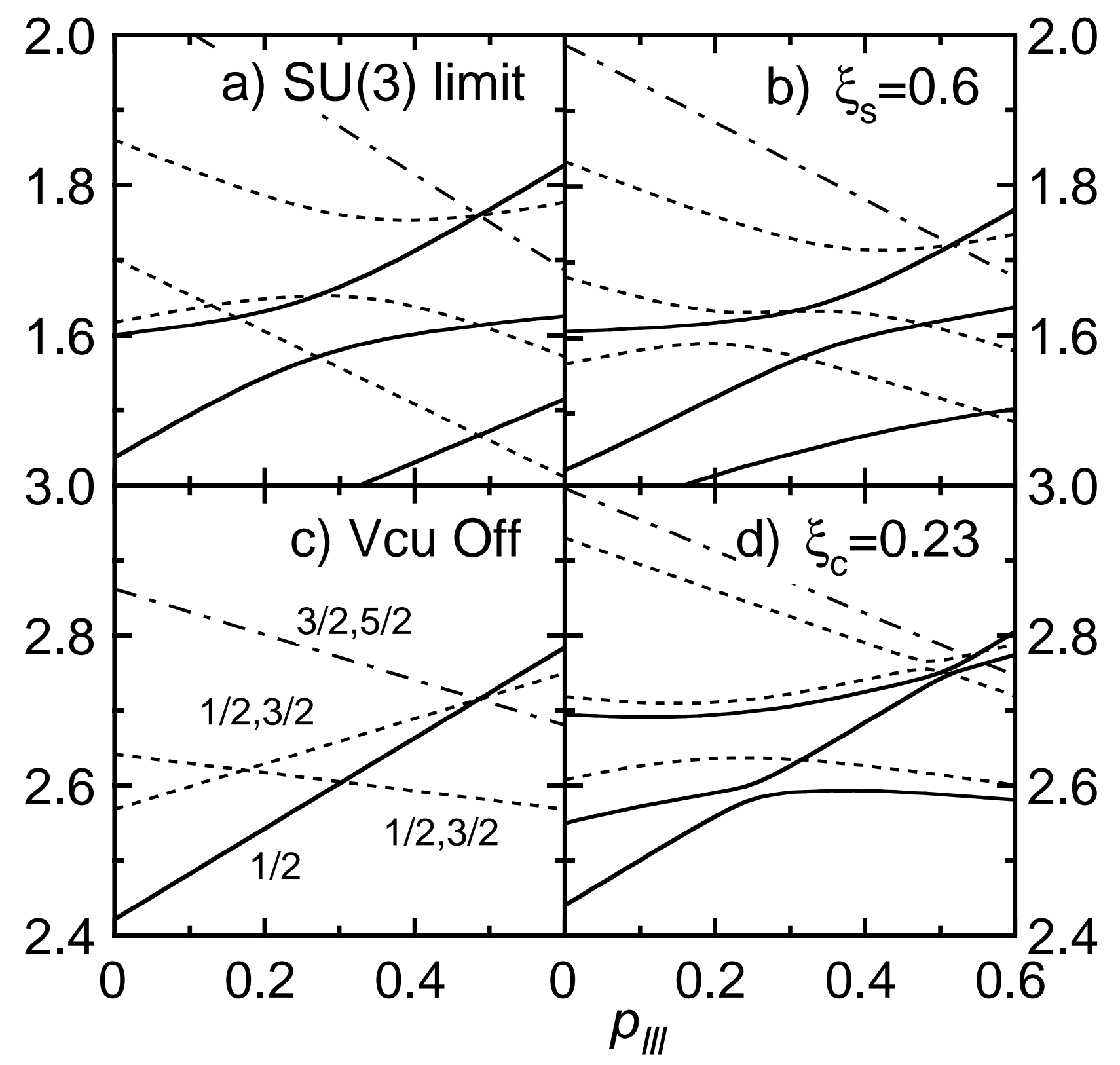

Fig 2 The $p_{\text {III }}$ dependence of the $P$-wave $\Lambda^{*}$ mass 